\title{
Total femoral replacement in non-neoplastic disorders
}

\begin{abstract}
Background: There are many non neoplastic disorders that can generate a significant deficit of femoral bone stock. In some cases, after repeated failed surgeries, this deficit may be so significant that standard reconstruction procedures and prosthesis are not useful. In these patients, it may be necessary to use a total femoral prosthesis to restore limb function.

Materials and methods: We have analyzed 6 patients with total femoral prosthesis with an average 3.8 year follow up.

Results: Four patients had a satisfactory outcome, regaining an independent ambulatory status, one died from septicaemia 6 weeks after surgery and another has persistent infection, a retained implant and remains on supressive antibiotic therapy.
\end{abstract}

Conclusion: Even if the number of evaluated patients is low, this analysis has revealed some important issues about surgical technique and indications of this procedure.
Volume 8 Issue 6 - 2017

\author{
Santiago Vedoya, Hernán del Sel, Germán \\ Garabano \\ Department of Orthopaedics and Traumatology, Buenos Aires \\ British Hospital
}

\begin{abstract}
Correspondence: Santiago Vedoya, Department of
Orthopaedics and Traumatology, Buenos Aires British Hospital, Perdriel 74 ( I209), CABA, Argentina, Tel (054II) 4304-I08I Emailspvedoya@gmail.com
\end{abstract}

Received: August 04, 2017| Published: September 07, 2017

Keywords: Total Femoral prosthesis, Femoral deficit, Non Neoplastic disorders

\section{Introduction}

The deficit of femoral bone stock is a frequent finding. This situation is mostly derived from the increased number of hip and knee arthroplasties and the consequent increase of failed implants, periprosthetic fractures and infections. There are other non-tumoral causes that generate this type of deficit, such as hardware failures in pathological bone, osteomielytis, congenital bone defects and metabolic bone diseases, etc. ${ }^{1-3}$

Several solutions have been proposed, which must be analyzed depending on the cause and magnitude of the femoral and acetabular bone loss, the patient's muscle status (particularly the gluteus medius), the presence of infection, the age and background of the patient, and, of course, the surgeon's expertise and his surgical facilities. This last aspect is essential, as revision and conversion surgeries are very demanding, for both patient and surgeon.

The most frequent alternatives include the use of cemented or non cemented long femoral stems, with proximal or distal fixation, associated or not to bone graft. Many studies have referred to the advantages and disadvantages of each of these treatment alternatives..$^{2-4}$ Nevertheless, in certain cases, the femoral bone deficit is so significant that there is not enough bone to properly fix any of these revision implants. In these patients, an option is the total hip replacement with a megaprosthesis, and among them, the total femur replacement (TFR). ${ }^{4-9}$

Buchman was the first to report a total femur replacement in a patient with Paget's disease10. The first total femur implants were made of hip and knee prosthesis united by a polyethylene shaft, to which these components were adapted. ${ }^{5,10}$ Even if this was a low cost versatile design, in time it was observed that polyethylene alone was not an adequate material to bear the load and torsions at a femoral level, particularly in young patients with high functional demand, since most of the initial patients were operated due to tumours. ${ }^{4}$ In the mid 80 s, modularity revolutionized reconstruction prosthesis, allowing the surgeon to estimate the existing bone defect, and therefore select the appropriate components for more accurate reconstructive surgery. ${ }^{1,11}$
The good outcome of patients treated with TFR for neoplastic causes widened the indication of this treatment to patients with massive femoral bone loss due to several non-neoplastic causes, not being the estimated survivorship of the patient an excluding factor in the decision process. ${ }^{12}$ However, except for the publications from Endo-Klinik in Hamburg, Germany, which exceed 100 cases. $^{7}$ the rest of TFR reports in non-neoplastic pathology analyze around 20 cases at the most. Additionally, in all these series the pre-operative diagnosis is varied, making it difficult to obtain from them a clear conclusion on the evolution of these patients on the medium and long term. In this study we evaluate the indications, surgical technique and post operative care of TFR in non-neoplastic disorders.

Table I Details of the 6 patients

\begin{tabular}{|c|c|c|c|c|c|c|}
\hline Sex & Age & Diagnosis & $\begin{array}{l}\mathbf{N}^{\circ} \text { of } \\
\text { previous } \\
\text { surgeries }\end{array}$ & $\begin{array}{l}\text { Follow } \\
\text { up } \\
\text { (years) }\end{array}$ & Complications & $\begin{array}{l}\text { Treatment of } \\
\text { complications }\end{array}$ \\
\hline$M$ & 71 & $\begin{array}{l}\text { Infected } \\
\text { periprosthetic } \\
\text { non-union }\end{array}$ & 11 & 3 & $\begin{array}{l}\text {-Persistence of } \\
\text { infection. } \\
\text {-Permanent } \\
\text { dislocation. }\end{array}$ & Surgical toilette. \\
\hline$F$ & 94 & $\begin{array}{l}\text { Periprosthetic } \\
\text { fracture }\end{array}$ & 3 & 4 & $\begin{array}{l}\text { Lateral popliteal } \\
\text { nerve injury. }\end{array}$ & Brace 6 months \\
\hline $\mathrm{F}$ & 82 & $\begin{array}{l}\text { Periprosthetic } \\
\text { non-union }\end{array}$ & 4 & 4 & $\begin{array}{l}\text { - Surgical injury of } \\
\text { Popliteal vein. } \\
\text {-Hematogenous late } \\
\text { infection. }\end{array}$ & $\begin{array}{l}\text { - By Pass } \\
\text { Surgical Toilette. }\end{array}$ \\
\hline $\mathrm{F}$ & 84 & $\begin{array}{l}\text { Periprosthetic } \\
\text { fracture }\end{array}$ & 3 & - & $\begin{array}{l}\text { Death due to sepsis } \\
45 \text { days } \\
\text { PO. }\end{array}$ & - \\
\hline $\mathrm{F}$ & 68 & $\begin{array}{l}\text { Failure of } \\
\text { revision due to } \\
\text { periprosthetic } \\
\text { fracture }\end{array}$ & 3 & 5 & 10 & - \\
\hline M & 82 & $\begin{array}{l}\text { Femoral non- } \\
\text { union in Paget } \\
\text { disease }\end{array}$ & 2 & 3 & - & - \\
\hline
\end{tabular}

\section{Materials and method}

A retrospective analysis of 6 patients (4 women and 2 men), 
operated between May 2014 and May 2016, was made (Table 1). The average age was 80 years old (68 to 94 years old). 4 patients were operated of their left limb.

The pre-operative diagnosis includes 5 patients $(84 \%)$ with periprosthetic fractures and non unions. Three patients with acute periprosthetic fractures in the short femoral segment lying between a long femoral stem and total knee prosthesis and 1 case each of periprosthetic non-union, infected periprosthetic non union and a twice operated non-union in a femur with Paget's disease and degenerative changes in the hip and knee (Figure 1).

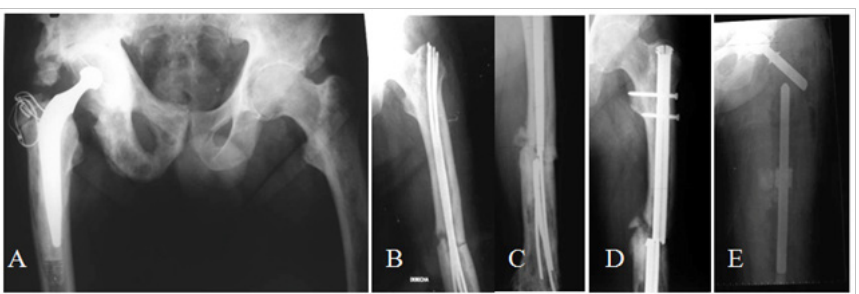

Figure I A- 82 year old patient with a well functioning right Charnley hip arthroplasty after 6 years, Paget's disease in both femurs and right hemipelvis, presents a left femur fracture. B- Osteosynthesis with Ender nails. C- Nails fail in 17 months. New osteosynthesis with locked nail. D- Failure in 8 months. E.TFR.

Before TFR surgery, 2 patients were able to barely walk a few steps with severe pain and assistance of a walking frame and the other 4 patients could not walk. All patients had had several previous surgeries, with an average of 4.3 surgeries (2 to 11).

The patients were evaluated at 20 and 45 days after surgery, and at 3, 6 and 12 months and annually.

\section{Preoperative planning}

Even if total femoral replacement surgery is technically less demanding that other complex reconstruction methods such as those using bone graft, it requires a meticulous pre-operative planning. ${ }^{5-7,13}$ $\mathrm{X}$-Ray measurements are extremely important., ${ }^{4,5,7} \mathrm{AP}$ and lateral views of both hips and the complete femur including the knee, must be obtained, with a scale allowing to evaluate the magnification, as the use of prosthesis which are custom made for each patient require to determine its dimensions in advance. It is important to bear in mind that frequently, the anatomic changes due to previous surgeries and bone loss, make measurement on the affected side inaccurate or impossible (Figure 2). In these cases, in order to determine limb and implant length and diameter, calculations on the opposite femur must be done, if it is not affected (Figure 2D).

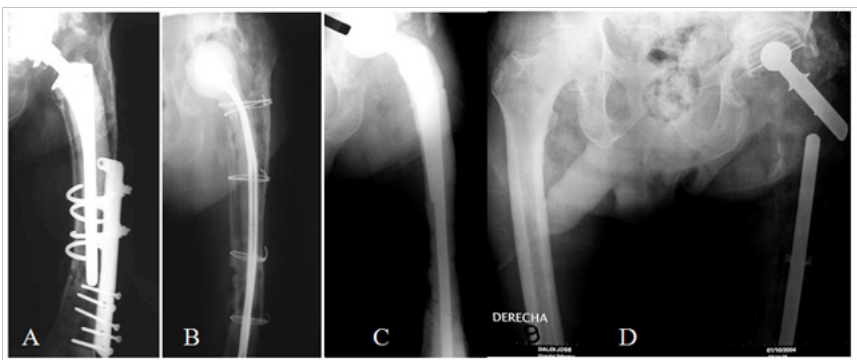

Figure 2 A- 7l year old patient with a chronically infected non-union of periprosthetic fracture. B- Initially treated with surgical toilette and cement spacer with antibiotics. C- In a second stage, it was necessary to resect almost all the remaining femur due to bone necrosis, and a new cement spacer with antibiotics was used. D- Finally, the patient received a TFR. The intact right femur can be used to make the pre-operative measurement.

Individual instruments and tools for removal of prosthetic material and/or the existing hardware must be anticipated, as well as the need for acetabular reconstruction, previous incisions that could modify the surgical approach or condition the post-operative recovery and any other relevant technical detail. In all cases infection must be ruled out by means of clinical, radiological, lab tests and the eventual joint aspiration for culture in suspicious cases. ${ }^{1}$

\section{Prosthesis characteristics}

In all patients, the Argentine custom made Fabroni megaprosthesis were used, which is manufactured by ROFA S.A. ${ }^{4-6}$ (Pje. Bella vista 1136 -C1416EIS- CABA, Argentina), with an approximate cost of 5.000 Euros. The prosthesis is composed by three parts. Its proximal segment is formed by two independent metallic components, the proximal stem and the femoral neck, both included in a high density polyethylene body. ${ }^{2,7,8}$ The distal femoral segment is also made of a high density polyethylene body with a cilindrical canal where the proximal stem is cemented, allowing for length regulation, and a distal constrained knee system. ${ }^{5,6}$ The third part is the rotational tibial component with a metallic distal stem (Figures 3-5).

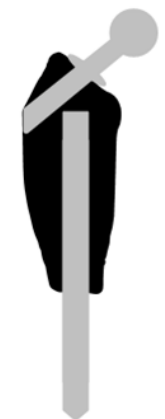

Steel

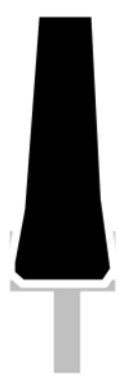

Polyethylene

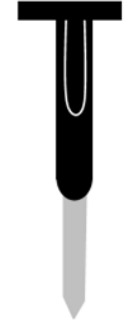

Figure 3 The three parts of the total femur prosthesis.

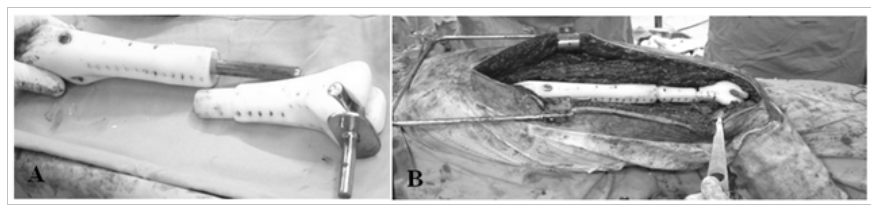

Figure 4 A- dissassembled total femoral megaprosthesis. B- Total femoral megaprosthesis assembled and in place.

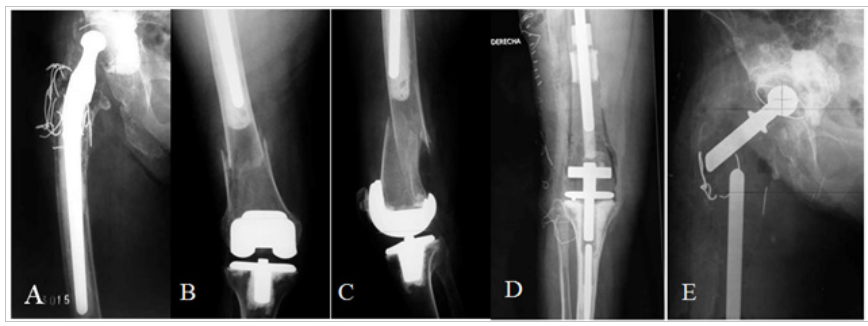

Figure 5 A, B y C- 94 year old lady with extremely osteopenic bone and a fracture in the short femoral shaft segment between a long stem hip prosthesis and a knee prosthesis. D- Post-operative X-ray, knee portion. EPost-operative X-ray, hip portion.

The high density polyethylene body has essential characteristics for the appropriate functioning of the prosthesis, such as absorbing physiological loads in order to avoid fatigue failures, filling dead space after the resection, preventing the formation of hematomas and providing anchorage for tendons and muscles by means of several 
holes in its surface. ${ }^{4-6}$ The metallic part of the implant is made of medical grade stainless steel AISI F 138. ${ }^{4-6}$

\section{Surgical technique}

The procedure has the following stages

A. The patient is laid in lateral or dorsal position, depending on the selected approach. A posterolateral or transtrochanteric approach can be used, depending on the type and number of previous surgeries, if there is or not a greater trochanter, the need of acetabular reconstruction and, of course, the surgeon's expertise. Notwithstanding which of these is selected, the approach must be extended laterally to reach the tibial tuberosity. A lateral knee arthrotomy is performed and the patella is dislocated medially, exercising cares not to avulse its distal insertion. Whenever possible, the main previous incision should be used. It is of upmost importance to preserve blood supply to the soft tissues in order to lower the risk of infection and to help the reconstruction during closure, assisting therefore in prosthetic stability and functional results. ${ }^{3,4,7-9,14}$

B. The Iliotibial band is divided longitudinally. ${ }^{4,7,8}$ In cases in which the muscle structures are conserved, the Gluteus Maximus and external rotators are identified, tagged for later reattachment and cut, being careful not to injury the Sciatic Nerve. The Vastus Lateralis and Gluteus Medius must be moved as one unit to the front. This step is essential in cases in which there is no Greater Trochanter, to facilitate abductor function. ${ }^{9}$ To minimize bleeding from the perforators, the linea aspera is better osteotomized rather than dissected. ${ }^{20}$ In cases of infection, a custom made mega antibiotic loaded cement spacer is used until reimplantation.

C. Acetabular reconstruction as necessary, cemented or cementless.

D. The tibial surface is prepared with a bone cut perpendicular to the tibial axis, approximately one centimeter from the joint surface, and the tibial component is then cemented.

E. The proximal and distal femoral components are assembled freely and the tibial constrained component is attached to the distal femur. The length of the implant is decided (Figure 4). This must be made checking stability, hip and knee motion, the balance between tension of the soft tissues and the range of motion at the knee and tension of the neurovascular structures. Once the appropriate position and length are defined, the assembly of the definitive prosthesis starts by cementing the metal shaft of the proximal femoral segment into the polyethylene canal of the distal femoral segment, with the desired length and 5 to 10 degrees of anteversion. ${ }^{1,4}$ (Figure 4). Then, the distal femur is reduced in the tibia and finally the hip is reduced, checking for stability and range of motion. ${ }^{14}$

F. Closure with soft tissue reconstruction is begun distally, with the consequent covering of the prosthesis with muscles if is possible. ${ }^{20}$ The muscle reconstruction requires special attention to guarantee a good prosthetic stability and to improve biomechanics and gait. ${ }^{1,3,9,14,15}$ It is preferable to close the capsule with non absorbable suture 1 since this provides immediate stability. When the capsule is appropriately sutured, the prosthesis should not dislocate..$^{1,10}$ It is essential to obtain an adequate muscle reinsertion in order not to suffer function loss and instability. ${ }^{1,4,16}$ The external rotator muscles are sutured to the holes that the prosthesis has for this purpose. If a portion of the Greater Trochanter still exists, it can be fixed to the prosthesis. ${ }^{6,15}$ Finally, the rest of the muscles can be reattached with non absorbable suture. ${ }^{15}$ Gluteus Maximus can be sutured to Vastus Lateralis..$^{4,7,10}$ or Fascia Lata. ${ }^{7}$ Both Gluteus Maximus and Psoas can be sutured slightly anterior, in order to give more lateral stability to the prosthesis. ${ }^{1,4,13,14}$ It is important to check the mobility of the limb to be sure that it is not limited by soft tissue tension. ${ }^{14}$ Careful hemostasis and minimizing dead space allow for better tissue healing. ${ }^{4,9}$ It is advisable to place drains for 48 to 72 hours and the use of a knee immovilizer during the first 4 to 8 weeks reduces the probability of dislocation..$^{1,4,12}$

During post-operative care, the suture of the abductors should be protected by partial weight bearing for 4 weeks, limited abduction and the use of walker or 2 canes not less than 8 weeks. This is essential to reduce limping and dislocations. ${ }^{1,12,13,16,17}$

\section{Results}

There were 2 failures. One patient died 45 days after surgery due to sepsis, and another patient, who had the TFR performed for a chronically infected periprosthetic non-union, failed due to persistence of infection. The remaining 4 patients can walk and are physically and emotionally satisfied with the outcome of surgery at an average follow-up of 3.8 years ( 3 to 5 years).

There were 2 major intraoperative complications. One patient sustained an injury to the femoral vein while it was being dissected away from fibrous tissue at the site of an infected distal femoral non union. This was treated with a by pass during the same procedure without further complications. Another patient developed a partial but definitive injury of the Lateral Popliteal nerve but walks well with 2 canes and an ankle brace.

There were 2 infections (33\%). One was due to the persistence of a previous process and is currently under suppressive antibiotic treatment, and the other corresponds to the patient who died due to sepsis 45 days after surgery. Another patient presented an hematogenous infection 2 years after surgery, which successfully evolved after a surgical toilette.

The only dislocation in this series (a chronic one), occurred in the patient with the persistence of infection. This patient currently requires 2 crutches to walk. The rest, 2 use a walker and 2 use only one cane, with 3 of 4 having a positive Trendelemburg sign.

\section{Discussion}

Even if the indications of TFR are extremely limited and specific, there is an increase in the number of patients in which this procedure offers an alternative to amputation or disarticulation..$^{15}$ This is due to the raising number of arthroplasties and their complications, to the longer life expectancy of the population and the development of effective mega-prosthesis for reconstruction.

The main objective of hip reconstruction surgery is to relieve pain and restore limb function (which in the cases of this study includes the ability to walk in most of the patients) by means of placing a durable and stable prosthesis. This is not easy to achieve in patients with a significant femoral bone deficit and, even more, if they have multiples comorbidities. ${ }^{4}$

We believe that TFR must be performed only in specialized centers and by surgeons with great expertise in hip and knee reconstruction surgeries, in order to optimize functional results and to reduce postoperative complications as much as possible.

This series does not have a number of patients big enough or a long follow up to obtain a comparable conclusion regarding the duration of the implant. Nevertheless, we consider this study useful to evaluate 
TFR indications in patients who cannot walk and to describe the details of surgical technique.

The indications of TFR in non neoplastic disorders can be diverse. We can mention femoral stem loosening, peri-prosthetic fractures, patients with multiple revisions, failed treatment of pathological fractures, chronic osteomyelitis, congenital bone defects or metabolic bone diseases. However, in this series, as well as in all the series reviewed, it is clear that the most frequent cause is femoral bone deficit associated to hip arthroplasty.

Even if TFR does not have a maximum age indication, it should be kept in mind that the older the patient is, it will be more difficult to regain the ability to walk. However, this does not mean a contraindication to treatment, considering that the alternative of an eventual amputation or desarticulation would surely confine these very elderly patients to bed or a wheelchair. ${ }^{14}$

A high rate of post-operative complications should be expected in this type of surgeries (up to $40 \%$ ), including, of course, a higher mortality rate compared to more conservative procedures.$^{4,7,8}$ This is due to the very large approach, the size of the implant, the high infection rates, the multiple previous surgeries, the clinical background of the patients and, in some cases, the pre-existing infection.

The most frequent complication is dislocation, which can reach $17 \%$ to $50 \%{ }^{1,4,7,12,18}$ There are several factors to consider regarding its incidence, but there is no doubt that the most significant of them is proper surgical technique. This includes a careful anatomic approach, the suture of the articular capsule, the reinsertion of the abductor muscles and the preservation of the acetabulum in the cases where a bipolar prosthesis can be used. ${ }^{1,12}$

The stability of the prosthesis can be optimized by the balance between the proximal traction of the abductors muscles and the Psoas muscle medially. As we previously mentioned, it can be sutured anteriorly to the capsule and serve as a reinforcement to it. ${ }^{1,4,18}$

Other essential aspects in order to achieve a stable prosthesis are: To appropriately place the prosthetic components (which can include the use of a constrained cup); to decide the length of the limb keeping in mind the muscle tension that gives stability to the hip above the length per se; a strict post-operative follow-up, eventually including the use of abduction devices or knee braces, and partial weight bearing for approximately 8 weeks. We believe that a knee immovilizer within the first 2 months after surgery must be routine in order to reduce dislocations. ${ }^{4}$ (prevents both flexion and adduction).

It should also be considered that there is an essential difference between patients operated for neoplastic causes and those operated for non-neoplastic ones regarding the dislocation rate, as the second group frequently has severe muscle deterioration, especially the abductors.

With the use of megaprosthesis, the infection rate can range between $3 \%$ and $21 \%$, with an increase both in early and in late infections. ${ }^{4,7,8,12,16}$ Even if multiples factors can cause these high infection rates, in our opinion the indication of this procedure must be carefully evaluated in patients with chronic infections and multiple previous surgeries, poor soft tissues or bad responses to previous surgeries. In these cases the failures rate grows abruptly, reaching 100 $\% .^{4}$

One of the failures of this series corresponds to a patient with these characteristics, whose infection process persisted in spite of two exhaustive toilettes, having placed 2 cemented mega-spacers with antibiotics, and aggressive antibiotic protocols.
Bearing in mind all these aspects, it is important to understand that age, previous comorbidities, post-operative physical demand and the patient's expectations must be carefully evaluated. However, even if it is an aggressive procedure, it has a very good emotional acceptance by patients. This is probably due to the fact that it relieves pain, combined with recovery of long lost walking ability and its good functional results.

Besides, even if it is a radical reconstruction method, it seems a valid alternative for treating the complication of arthroplasties (generally of the hip although also of the knee) where other solutions apart from amputation would imply long bed rest periods. ${ }^{7,19}$

Total femoral replacement restores the femoral integrity, allowing the patient to re-assume walking with minimum or acceptable pain levels, giving him back his independence. This situation is clearly superior to the surgical alternatives, such as amputation or disarticulation. ${ }^{15,20}$ and this sole fact justifies the risk of performing a procedure so demanding both for patient and surgeon.

\section{Acknowledgments}

None.

\section{Conflicts of interest}

None.

\section{References}

1. Bickels J, Meller I Reconstruction of hip stability alter proximal and total fémur resections. Clin Orthop Relat res. 2000;375:218-230.

2. Parvizi J, Sim F Proximal Femoral Replacements with Megaprosteses. Clin Orthop Relat res. 2004;420:169-175.

3. Sim FH, Chao EY Segmental prosthetic replacement of the hip and knee: Tumor Prostheses for bone and Joint Reconstruction. Thieme-Stratton, pp. 1983;247-266.

4. Del Sel H, Vedoya S, Garabano G Reemplazo de cadera con megaprótesis en patología no tumoral.Revista de la AAOT: 2009;74(3):282-289.

5. Benetti A, Fabroni $H$ Endoprotesis no convencional: técnica de reemplazo total de fémur Bol trab soc. Argentina de Ortopedia $y$ Traumatologia. 1976;41:291-296.

6. Fabroni RH1, Castagno A, Aguilera AL et al. Long Term Results of Limb Salvage with the Fabroni Custom Made Endoprosthesis. Clin Orthop Relat Res. 1999;358:41-52.

7. Fountain JR, Dalby-Ball J, Carroll FA et al. The use of Tota Femoral Arthroplasty as a Limb Salvage Procedure. J Arthrop. 2007;22(5):663-669.

8. Friesecke C, Plutat J, Block A Revision Arthroplasty with use of a Total Femur Prosthesis. JBJS. 2005;87(12):2693-2701.

9. Morris H, Capanna R Modular endoprosthetic replacement alter total resection of the fémur for malignant tumor. Int Orthop. 1994;18:90-95.

10. Buchman J Total Femur and Knee joint replacement with a vitallium endoprosthesis. Bull Hosp Joint Dis. 1965;26:21-34.

11. Chao EY A composite fixation principle for modular segmental defect replacement (SDR) protheses. Orthop Clin North Am. 1989;20(3):439-453.

12. Ahlmann ER, Menendez LR, Kermani C et al. Survivorship and clinical outcome of modular endoprosthetic reconstruction for neoplastic disease of the lower limb. JBJS. 2006;88(6):790-795.

13. Ozaki T, Kaneko S, Kunisada T, Kawai A, Dan'ura T, et al. Reconstruction of the hip abductors after resection of the proximal fémur. Int Orthop. 1999;23(3):182-183. 
14. Ward W, Dorey F Total Femoral Endoprosthetic Reconstruction. Clin Orthop Relat Res. 1995;316:195-206.

15. Steinbrink K, Engelbretch E The Total Femoral Prosthesis. A preliminary report. JBJS. 1982;64(3):305-312.

16. Giurea A, Paternostro T Function of reinserted abductor muscles after femoral replacement. JBJS. 1998;80(2):284-287.

17. bNerubay J, Katznelson A Total femoral replacement. Clin Orthop Relat Res. 1988;229:143-148.
18. Johnsson R, Carlsson A, Kisch K et al. Function following mega total hip arthroplasty compared with convencional total hip arthroplasty and healthy matched controls. Clin Orthop Relat Res. 1985;192:159-167.

19. Bhattacharyya T, Chang D Mortality after periprosthetic fracture of the femur. JBJS 2007;89(12):2658-2662.

20. Present D, Kuschner S Total femoral replacement. A case report with 35 years follow up study. Clin Orthop Relat Res. 1990;251:166. 